\begin{tabular}{|c|c|c|c|c|c|c|}
\hline \multirow{4}{*}{ Impact Factor: } & ISRA (India) & $=3.117$ & SIS (USA) & $=0.912$ & ICV (Poland) & $=6.630$ \\
\hline & ISI (Dubai, UAI & $=0.829$ & РИНЦ (Russia) & $=0.156$ & PIF (India) & $=1.940$ \\
\hline & GIF (Australia) & $=0.564$ & ESJI (KZ) & $=8.716$ & IBI (India) & $=4.260$ \\
\hline & JIF & $=1.500$ & SJIF (Morocco & $=5.667$ & OAJI (USA) & $=0.350$ \\
\hline
\end{tabular}

\section{SOI: $1.1 /$ TAS $\quad$ DOI: $10.15863 /$ TAS \\ International Scientific Journal Theoretical \& Applied Science}

p-ISSN: 2308-4944 (print) e-ISSN: 2409-0085 (online)

Year: $2019 \quad$ Issue: $05 \quad$ Volume: 73

Published: $10.05 .2019 \quad \underline{\text { http://T-Science.org }}$

SECTION 2. Applied mathematics.

Mathematical modeling.
QR - Issue

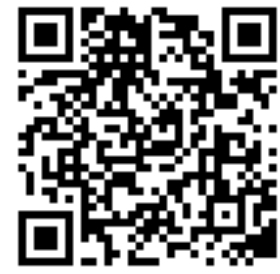

Taraz State University named after M.Kh. Dulati candidate of technical sciences, member of PILA (USA), department of «Mathematics», deputy director on Science of faculty of Information technologies, automation and telecommunications, Taraz State University named after M.Kh. Dulati Shev_AlexXXXX@mail.ru
Alexandr Shevtsov

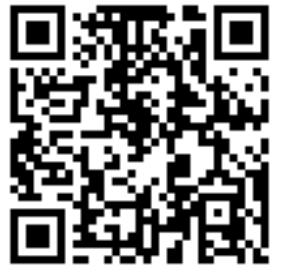

Didarkhan Utemgaliev master student,

\title{
THE DEVELOPMENT OF THE LIBRARY IN MAPLE IMPLEMENTS THE ALGORITHMS OF THE METHOD OF INTERIOR POINT
}

Abstract: In this paper, we develop a library for Maple implementing algorithms for applying the method of internal points.

Key words: Maple, interior point, library.

Language: English

Citation: Utemgaliev, D., \& Shevtsov, A. (2019). The development of the library in Maple implements the algorithms of the method of interior point. ISJ Theoretical \& Applied Science, 05 (73), 266-272.

Soi: http://s-o-i.org/1.1/TAS-05-73-37 Doi: crossef https://dx.doi.org/10.15863/TAS.2019.05.73.37

\section{Introduction}

The created library will be based on the previously used algorithms for the method of internal points. The name of the library InteriorPointUtemgaliev.MapleLib.

Set the names of algorithms:

- IP Plot

$\operatorname{read}($ 'D: InteriorPointUtemgaliev.MapleLib') :

with (InteriorPoint);

[IP_L3,IP_OptimalAllocation, IP_Plot, IP_Slack,IP_Slack_new]

Library code:

restart:

InteriorPoint $:=$ table () :

InteriorPoint $[$ IP Plot $]:=\operatorname{proc}($ Equation, $x 1, x 2)$ local $n$ :

with ( plots) :

plot $3 d($ Equation, $x[1]=x 1, x[2]=x 2$, shading $=$ zhue, axes $=$ boxed, style

$=$ patchcontour, contours $=10)$;

end proc:

Code of the main program:
- IP_OptimalAllocation

- IP_Slack

- IP_Slack_new

- IP_L3

Connection of the library is carried out by the command 


\begin{tabular}{|c|c|c|c|c|c|c|}
\hline \multirow{4}{*}{ Impact Factor: } & ISRA (India) & $=3.117$ & SIS (USA) & $=0.912$ & ICV (Poland) & $=6.630$ \\
\hline & ISI (Dubai, UAE & $=0.829$ & РИНЦ (Russia) & $=0.156$ & PIF (India) & $=1.940$ \\
\hline & GIF (Australia) & $=0.564$ & ESJI (KZ) & $=8.716$ & IBI (India) & $=4.260$ \\
\hline & JIF & $=1.500$ & SJIF (Morocco) & $=5.667$ & OAJI (USA) & $=0.350$ \\
\hline
\end{tabular}

restart;

read( 'D: InteriorPointUtemgaliev.MapleLib') :

with (InteriorPoint);

$$
\text { [IP_OptimalAllocation, IP_Plot }]
$$

Наша целевая функции приведена в виде параболоида:

$$
\begin{aligned}
& F:=100 \cdot x[1] \cdot x[2]^{*} \exp \left(\frac{-x[1]^{\wedge} 2}{3}-\frac{x[2]^{\wedge} 2}{3}\right) ; \\
& I P \_P l o t(F, 0 . .4,0 . .4) ; \\
& F:=100 x_{1} x_{2} \mathrm{e}^{-\frac{x_{1}^{2}}{3}-\frac{x_{2}^{2}}{3}}
\end{aligned}
$$

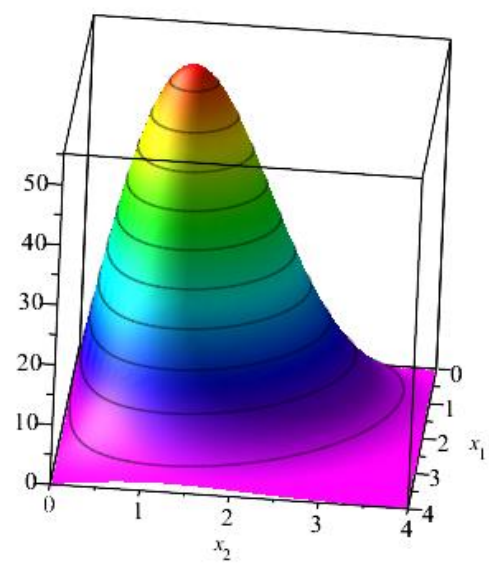

Library code:

InteriorPoint $[$ IP_OptimalAllocation $]:=\operatorname{proc}(o b, c o n 1$, con 2$)$ local $F, x x 1, x x 2$, myl, my2, my3, my4, my5:

with (plots) :

$x x 1:=$ evalf $($ solve $($ solve $($ convert $($ conl, equality),$x[2])=\operatorname{solve}(\operatorname{convert}($ con 2 ,

equality), $x[2]), x[1]), 5)$ :

$x \times 2:=\operatorname{eval}($ solve( convert (conl, equality), $x[2]), x[1]=x x 1)$ :

$m y l:=\operatorname{plot}($ solve $($ convert $($ conl, equality $), x[2]), x[1]=0 . .10$, color $=$ red, thickness $=2)$ :

my $2:=\operatorname{plot}($ solve $($ convert $(\operatorname{con} 2$, equality $), x[2]), x[1]=0 . .10$, color $=$ green,

$$
\text { thickness }=2 \text { ) : }
$$

my $3:=$ plots:-contourplot $(o b, x[1]=0 . .5, x[2]=0 . .5$, color $=$ black, contours $=10)$; my $4:=$ plots:-pointplot $(\{[x x 1, x x 2]\}$, axes $=$ boxed, symbol $=$ solidcircle, symbolsize $=20)$ :

my $5:=$ plots:-textplot $([x x 1, x x 2$, "Optimal Allocation" $]$, align $=\{A B O V E, R I G H T\}$, font $=[$ times, bold, 16]) :

$\operatorname{print}([\operatorname{eval}(o b,\{x[1]=x x 1, x[2]=x x 2\}), x[1]=x x 1, x[2]=x x 2])$; plots:-display $(\{m y 1, m y 2, m y 3, m y 4, m y 5\}$, view $=[0 . .4,0 . .5])$;

end proc:

Code of the main program: 


\begin{tabular}{llllll} 
& ISRA (India) $=\mathbf{3 . 1 1 7}$ & SIS (USA) & $=\mathbf{0 . 9 1 2}$ & ICV (Poland) & $=\mathbf{6 . 6 3 0}$ \\
Impact Factor: & ISI (Dubai, UAE) $=\mathbf{0 . 8 2 9}$ & PUHL (Russia) $=\mathbf{0 . 1 5 6}$ & PIF (India) & $=\mathbf{1 . 9 4 0}$ \\
& GIF (Australia) $=\mathbf{0 . 5 6 4}$ & ESJI (KZ) & $=\mathbf{8 . 7 1 6}$ & IBI (India) & $=\mathbf{4 . 2 6 0}$ \\
& JIF & $\mathbf{1 . 5 0 0}$ & SJIF (Morocco) $=\mathbf{5 . 6 6 7}$ & OAJI (USA) & $\mathbf{0 . 3 5 0}$ \\
\hline
\end{tabular}

Теперь мы можем построить такую целевую функцию с соответствующими ограничениями и найти оптимальное решение следующим образом:

restart;

$\operatorname{read}\left({ }^{\prime} D\right.$ : InteriorPointUtemgaliev.MapleLib') :

with (InteriorPoint);

$F:=100 \cdot x[1] \cdot x[2]^{*} \exp \left(\frac{-x[1]^{\wedge} 2}{3}-\frac{x[2]^{\wedge} 2}{3}\right):$

ogr_l $1:=4 \cdot x[1]+5 \cdot x[2] \leq 20$ :

ogr $2:=6 \cdot x[1]+1 \cdot x[2] \leq 18$ :

IP_OptimalAllocation $\left(F, o g r_{-} 1, o g r_{-} 2\right)$;

$$
\begin{gathered}
{[\text { IP_OptimalAllocation, IP_Plot, IP_Slack }]} \\
{\left[14.24508347, x_{1}=2.6923, x_{2}=1.846160000\right]}
\end{gathered}
$$

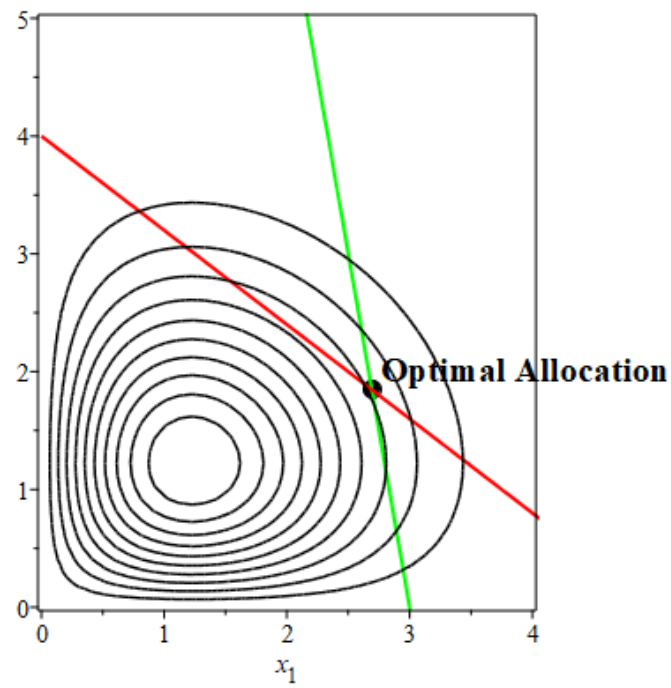

Library code:

InteriorPoint $[$ IP_Slack] := proc(ob,con1, con2) local $F, x x 1, x x 2, m y 1, m y 2$, my3, my4, my5, Slack1, Slack2, L:

$x x l:=\operatorname{evalf}($ solve (solve (convert (conl, equality),$x[2])=\operatorname{solve}($ convert (con2, equality), $x[2]), x[1]), 5)$ :

$x \times 2:=\operatorname{eval}($ solve (convert(conl, equality), $x[2]), x[1]=x x 1)$ :

$m y 1:=\operatorname{plot}($ solve $($ convert $($ conl, equality $), x[2]), x[1]=0 . .10$, color $=$ red, thickness $=2)$ :

$m y 2:=\operatorname{plot}($ solve $($ convert (con2, equality),$x[2]), x[1]=0 . .10$, color $=$ green, thickness $=2)$ :

Slackl := rhs (conl) - lhs $(\operatorname{con} 1):$

Slack $2:=r h s(\operatorname{con} 2)-\operatorname{lhs}(\operatorname{con} 2):$

$L:=o b-\lambda \cdot(\ln ($ Slack 1$)+\ln ($ Slack 2$)):$

$m y 3:=$ plots:-contourplot $($ eval $(L, \lambda=0), x[1]=0 . .10, x[2]=0 . .10$, color $=$ black, contours $=10)$ :

$\operatorname{print}($ Slack1);print $($ Slack2); $\operatorname{print}(L)$;

$\operatorname{print}(m y 3)$;

$\operatorname{print}($ plots:-display $(\{m y 1, m y 2, m y 3\}$, view $=[0 . .4,0 . .5]))$;

end proc:

Code of the main program: 


\begin{tabular}{|c|c|c|c|c|c|c|}
\hline \multirow{4}{*}{ Impact Factor: } & ISRA (India) & $=3.117$ & SIS (USA) & $=0.912$ & ICV (Poland) & $=6.630$ \\
\hline & ISI (Dubai, UAE & $=0.829$ & РИНЦ (Russia & $=0.156$ & PIF (India) & $=1.940$ \\
\hline & GIF (Australia) & $=0.564$ & ESJI (KZ) & $=8.716$ & IBI (India) & $=4.260$ \\
\hline & JIF & $=1.500$ & SJIF (Morocco & $=5.667$ & OAJI (USA) & $=0.350$ \\
\hline
\end{tabular}

Теперь решим вышеуказанную проблему, используя метод внутренней точки

Введем новую переменную slack

$$
y \geq 0
$$

неравенство

$$
A \cdot x \leq b
$$

можно преобразовать в форму

$$
A \cdot x+y=b
$$

Это означает, что наши ограничения могут быть записаны как:

$4 \cdot x[1]+5 \cdot x[2]+20$;

$6 \cdot x[1]+1 \cdot x[2]+10$

$$
\begin{gathered}
4 x_{1}+5 x_{2}+20 \\
6 x_{1}+x_{2}+10
\end{gathered}
$$

$4 \cdot x[1]+5 \cdot x[2]+y=20$;

$6 \cdot x[1]+1 \cdot x[2]+y=10$;

$$
\begin{gathered}
4 x_{1}+5 x_{2}+y=20 \\
6 x_{1}+x_{2}+y=10
\end{gathered}
$$

restart;

$\operatorname{read}\left({ }^{\prime} D\right.$ : InteriorPointUtemgaliev.MapleLib') :

with (InteriorPoint);

$F:=100 \cdot x[1] \cdot x[2] * \exp \left(\frac{-x[1]^{\wedge} 2}{3}-\frac{x[2]^{\wedge} 2}{3}\right):$

ogr_l $:=4 \cdot x[1]+5 \cdot x[2] \leq 20:$

ogr_2 $:=6 \cdot x[1]+1 \cdot x[2] \leq 10$ :

IP_Slack $(F$, ogr_l,ogr_2);

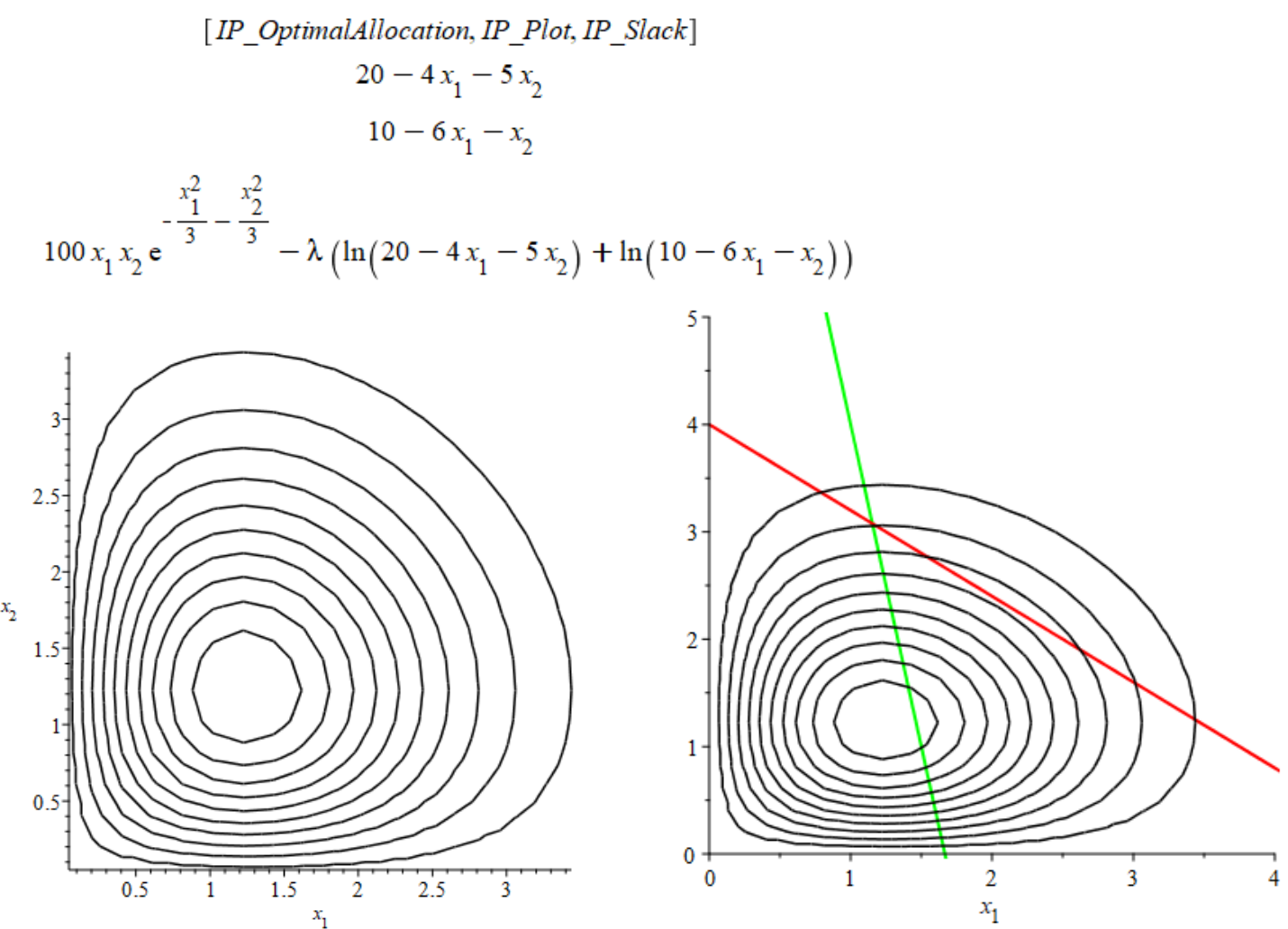

Код библиотеки: 


\begin{tabular}{|c|c|c|c|c|c|c|}
\hline \multirow{4}{*}{ Impact Factor: } & ISRA (India) & $=3.117$ & SIS (USA) & $=0.912$ & ICV (Poland) & $=6.630$ \\
\hline & ISI (Dubai, UAE & $=0.829$ & РИНЦ (Russia & $=0.156$ & PIF (India) & $=1.940$ \\
\hline & GIF (Australia) & $=0.564$ & ESJI (KZ) & $=8.716$ & IBI (India) & $=4.260$ \\
\hline & JIF & $=1.500$ & SJIF (Morocco & $=5.667$ & OAJI (USA) & $=0.350$ \\
\hline
\end{tabular}

InteriorPoint $\left[I P \_S l a c k \_n e w\right]:=\operatorname{proc}(o b, c o n 1$, con2) local $F, x x 1, x x 2$, myl, my2, my3, my4, my5, Slack $\overline{1}$, Slack2, L_new:

$x x 1:=\operatorname{evalf}($ solve (solve (convert (conl, equality), $x[2])=\operatorname{solve}(\operatorname{convert}(\operatorname{con} 2$, equality), $x[2]), x[1]), 5)$ :

$x \times 2:=\operatorname{eval}($ solve (convert (conl, equality), $x[2]), x[1]=x x 1):$

$m y 1:=\operatorname{plot}($ solve $($ convert $($ conl, equality $), x[2]), x[1]=0 . .10$, color $=$ red, thickness

$=2)$ :

my $2:=\operatorname{plot}($ solve $($ convert $($ con 2, equality $), x[2]), x[1]=0 . .10$, color $=$ green,

thickness $=2$ ) :

Slackl $:=\operatorname{rhs}(\operatorname{con} 1)-\operatorname{lhs}(\operatorname{con} 1):$

Slack $2:=\operatorname{rhs}(\operatorname{con} 2)-\operatorname{lhs}(\operatorname{con} 2):$

$L_{-}$new $:=\frac{(-\operatorname{lhs}(\mathrm{con} 1)-\operatorname{lh} s(\mathrm{con} 2))}{2}-\lambda \cdot(\operatorname{rhs}(\operatorname{con} 1)-\log (\operatorname{lh} s(\mathrm{con} 1))$

$+\log ($ Slack2) ) :

print $\left(L_{-}\right.$new $)$;

$m y 3:=$ plots:-contourplot $\left(\right.$ eval $\left(L_{-}\right.$new,$\left.\lambda=0\right), x[1]=0 . .50, x[2]=0 . .50$, color

= black, contours $=10)$ :

print (my3);

$\operatorname{print}($ plots:-display $(\{m y 1, m y 2, m y 3\}))$;

end proc:

Code of the main program:

restart;

read( 'D: InteriorPointUtemgaliev.MapleLib') :

with (InteriorPoint);

$F:=100 \cdot x[1] \cdot x[2]^{*} \exp \left(\frac{-x[1]^{\wedge} 2}{3}-\frac{x[2]^{\wedge} 2}{3}\right):$

ogr_l $:=4 \cdot x[1]+5 \cdot x[2] \leq 20$ :

ogr_2 $:=6 \cdot x[1]+1 \cdot x[2] \leq 10$

IP_Slack_new $\left(F, o g r \_1, o g r \_2\right)$;

[IP_Optimalallocation, IP_Plot, IP_Slack, IP_Slack_new] $-5 x_{1}-3 x_{2}-\lambda\left(20-\ln \left(4 x_{1}+5 x_{2}\right)+\ln \left(10-6 x_{1}-x_{2}\right)\right)$
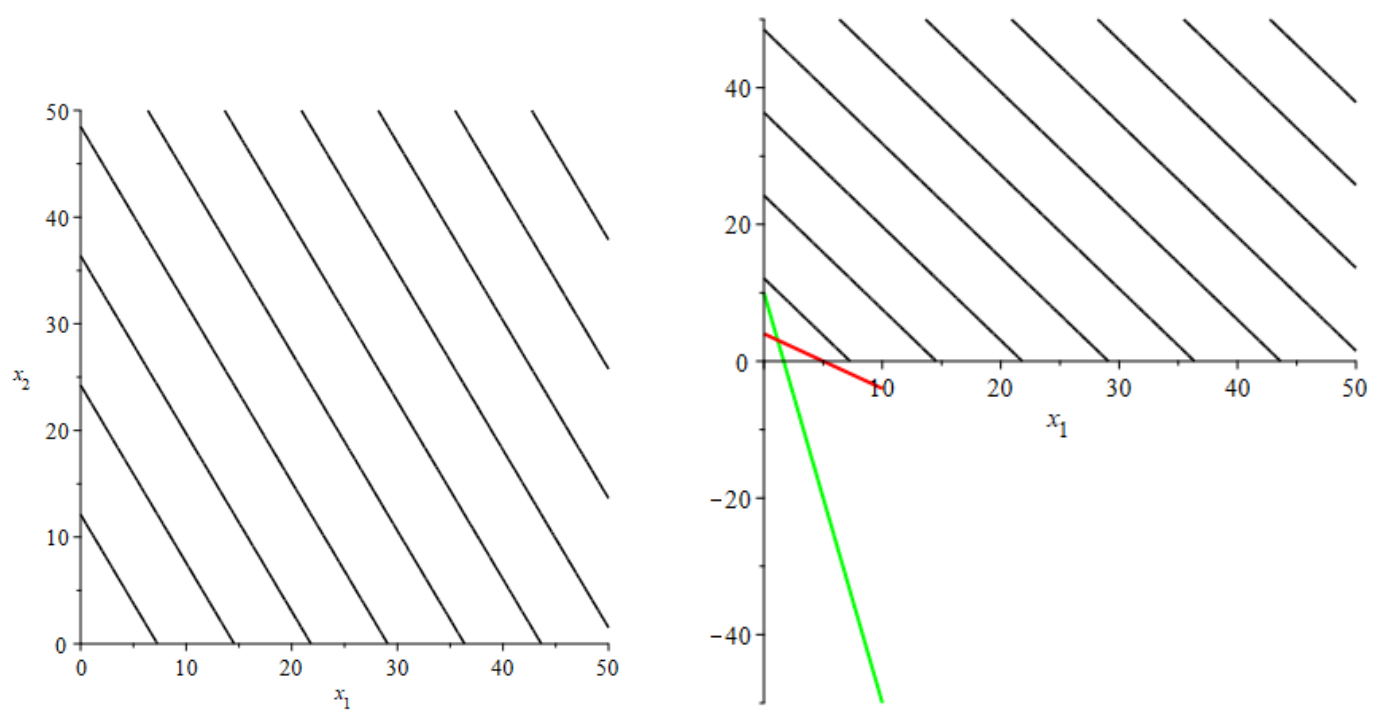

Library code: 


\begin{tabular}{llllll} 
& ISRA (India) $=\mathbf{3 . 1 1 7}$ & SIS (USA) & $=\mathbf{0 . 9 1 2}$ & ICV (Poland) & $=\mathbf{6 . 6 3 0}$ \\
Impact Factor: & ISI (Dubai, UAE) $=\mathbf{0 . 8 2 9}$ & PUHL (Russia) $=\mathbf{0 . 1 5 6}$ & PIF (India) & $=\mathbf{1 . 9 4 0}$ \\
& GIF (Australia) $=\mathbf{0 . 5 6 4}$ & ESJI (KZ) & $=\mathbf{8 . 7 1 6}$ & IBI (India) & $=\mathbf{4 . 2 6 0}$ \\
& JIF & $\mathbf{1 . 5 0 0}$ & SJIF (Morocco) $=\mathbf{5 . 6 6 7}$ & OAJI (USA) & $\mathbf{0 . 3 5 0}$ \\
\hline
\end{tabular}

InteriorPoint $\left[I P \_L 3\right]:=\operatorname{proc}(o b, \lambda 0$, conl, con2, con3,og $)$ local my0, my1, my2, my3:

my $0:=\operatorname{plot}($ solve $($ convert (conl, equality),$x[2]), x[1]=o g$, color $=$ red, thickness

$=2)$ :

$m y 1:=\operatorname{plot}($ solve $($ convert $($ con 2, equality $), x[2]), x[1]=o g$, color = green, thickness

$=2)$ :

$m y 2:=\operatorname{plot}($ solve $($ convert $($ con3, equality $), x[2]), x[1]=$ og, color $=$ blue, thickness

$=2)$ :

$m y 3:=$ plots:-contourplot $(\operatorname{eval}(o b, \lambda=\lambda 0), x[1]=o g, x[2]=o g$, color $=$ black,

contours $=50)$ :

$\operatorname{print}($ plots:-display $(\{m y 0, m y 1, m y 2, m y 3\}$, view $=[\circ g, o g]))$;

end proc:

Code of the main program:

restart;

$\operatorname{read}\left({ }^{\prime} D\right.$ : InteriorPointUtemgaliev.MapleLib') :

with (InteriorPoint);

$F:=x[1]+x[2]-\lambda \cdot(\ln (x[1]+2 \cdot x[2]-100)+\ln (x[1])+\log (x[2])):$

$o g r_{-} 1:=-x[1]-2 \cdot x[2]+100 \leq 0$ :

ogr_2 $:=-14 x[1]-8 \cdot x[2]+950 \leq 0$ :

$o g r_{-} 3:=-9 x[1]-4 \cdot x[2]+620 \leq 0$ :

$\lambda 0:=0:$

$I P \_L 3\left(F, \lambda 0, o g r_{-} 1, o g r_{-} 2, o g r_{-} 3,-30 . .200\right)$;

$\lambda \overline{0}:=0.1$ :

IP_L3 $\left(F, \lambda 0, o g r_{-} 1, o g r_{-} 2, o g r_{-} 3,0 . .150\right)$;

[IP_L3,IP_OptimalAllocation, IP_Plot, IP_Slack, IP_Slack_new]
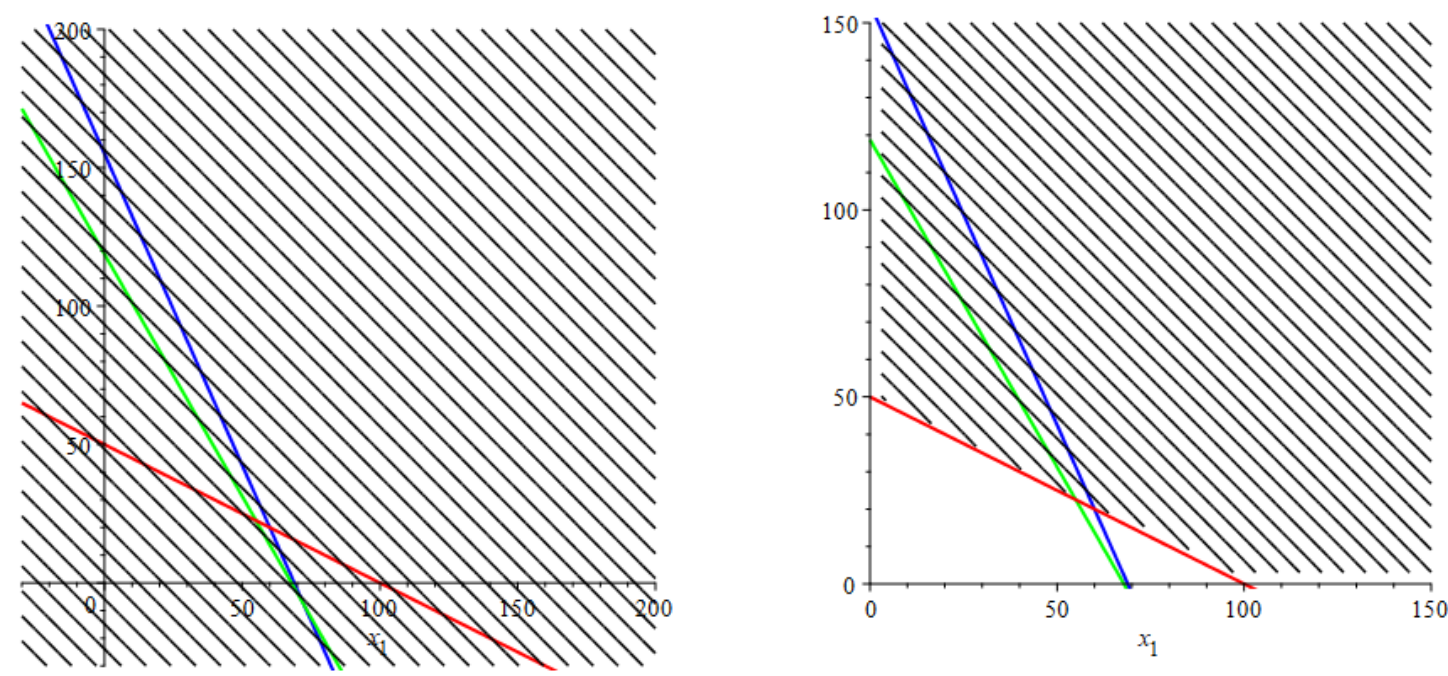

The library is compiled and saved by the command:

save(InteriorPoint, 'D: InteriorPointUtemgaliev.MapleLib') :

\section{Conclusion}

As a result of the study, a review of the main methods of mathematical optimization for problems with constraints: the method of internal points, the problem of mathematical optimization, simplex method, projective gradient descent, ellipsoid method, the method of internal point, the problem of quadratic programming, the theoretical foundations of linear optimization. Algorithms of application of methods of internal points in Maple are considered. The existing methods used by the Maple optimization package are studied. Algorithms interior point method for Maple. Developed the library in Maple implements the algorithms of the method of interior point. The created library has been tested and can be used in solving optimization problems by the method of internal points. 


\begin{tabular}{|c|c|c|c|c|c|c|}
\hline \multirow{4}{*}{ Impact Factor: } & ISRA (India) & $=3.117$ & SIS (USA) & $=0.912$ & ICV (Poland) & $=6.630$ \\
\hline & ISI (Dubai, UAE & $=0.829$ & РИНЦ (Russia & $=0.156$ & PIF (India) & $=1.940$ \\
\hline & GIF (Australia) & $=0.564$ & ESJI (KZ) & $=8.716$ & IBI (India) & $=4.260$ \\
\hline & JIF & $=1.500$ & SJIF (Morocce & $=5.667$ & OAJI (USA) & $=0.350$ \\
\hline
\end{tabular}

\section{References:}

1. Dantzig, G. B. (2003). Linear Programming 2: Theory and Extensions. Springer-Verlag.

2. Chernikov, Y. (n.d.). Sistemnyy analiz $i$ issledovanie operatsiy.

3. Bonnans, J. F., et al. (2006). Numerical optimization: Theoretical and practical aspects. Second revised ed. of translation of 1997 French. Berlin: Springer-Verlag, 2006. p.490. ISBN 3540-35445-X. DOI:10.1007/978-3-540-354475.

4. Karmarkar, N. (1984). Proceedings of the sixteenth annual ACM symposium on Theory of computing - STOC '84" (PDF): 302. DOI:10.1145/800057.808695. ISBN 0-89791133-4.
5. Mehrotra, S. (1992). On the Implementation of a Primal-Dual Interior Point Method. SIAM Journal on Optimization, 2 (4): 575. DOI:10.1137/0802028.

6. Nocedal, J. (1999). Numerical Optimization. New York, NY: Springer. ISBN 0-387-98793-2.

7. Press, W. H., et al. (2007). Section 10.11. Linear Programming: Interior-Point Methods // Numerical Recipes: The Art of Scientific Computing. 3rd. Cambridge University Press. ISBN 978-0-521-88068-8.

8. Wright, S. (1997). Primal-Dual Interior-Point Methods. Philadelphia, PA: SIAM. ISBN 089871-382-X.

9. Boyd, S. (2004). Convex Optimization. Cambridge University Press. 